Supplement of Atmos. Meas. Tech. Discuss., 8, 3697-3728, 2015

http://www.atmos-meas-tech-discuss.net/8/3697/2015/

doi:10.5194/amtd-8-3697-2015-supplement

(C) Author(s) 2015. CC Attribution 3.0 License.

(c) (i)

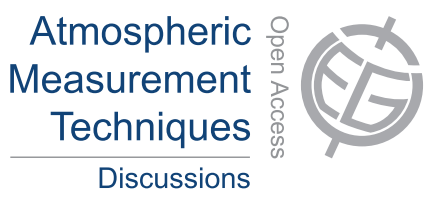

Supplement of

\title{
Relative drifts and biases between six ozone limb satellite measurements from the last decade
}

\section{N. Rahpoe et al.}

Correspondence to: N. Rahpoe (nabiz@iup.physik.uni-bremen.de) 
Contour plots of derived parameters: relativedrift $\alpha$ and relative bias $\beta$ from the multi-regression model are shown herefor all pair combinations and latitude bands.

Following pairs of comparison/reference sensors are included here, as shown in Table 1. The differences in contour plots are shown as Comp. - Ref. (see Fig. 1 - Fig. 15). In the contour plots the areas of statistically significant values at confidence interval $\mathrm{CI}=95 \%$, i.e., $(\alpha, \beta)>2 \sigma_{(\alpha, \beta)}$ are marked with black contour lines.

Table 1: Pairwise combinations of comparison sensor (Comp.) and reference sensor (Ref.).

\begin{tabular}{lcccccc}
\hline Comp./Ref. & SCIAMACHY & GOMOS & OSIRIS & MIPAS & ACE-FTS & SMR \\
\hline \hline SCIAMACHY & - & - & - & - & - & - \\
GOMOS & Y & - & - & - & - & - \\
OSIRIS & Y & Y & - & - & - & - \\
MIPAS & Y & Y & Y & - & - & - \\
ACE-FTS & Y & Y & Y & Y & - & - \\
SMR & Y & Y & Y & Y & Y & - \\
\hline
\end{tabular}


Relative Bias distribution GOMOS V6 - SCIA V2.5 2002 - 2011

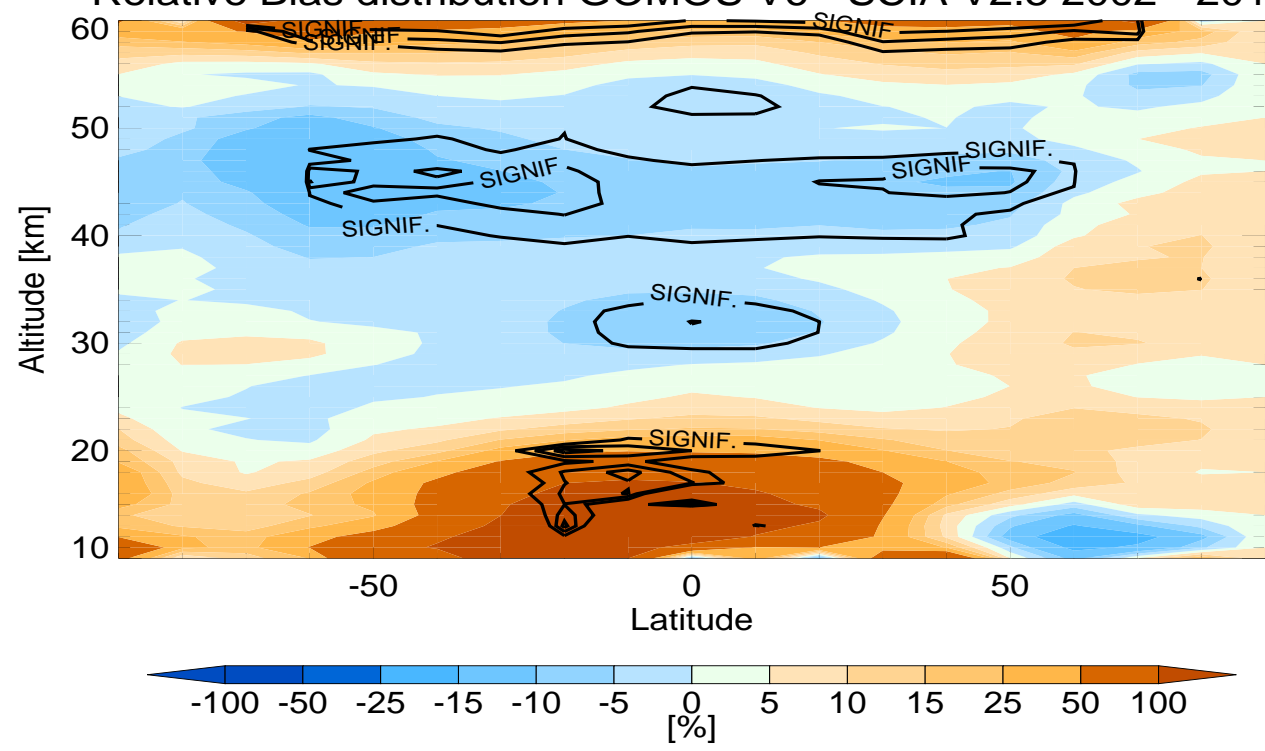

Relative Drift distribution GOMOS V6 - SCIA V2.5 2002 - 2011

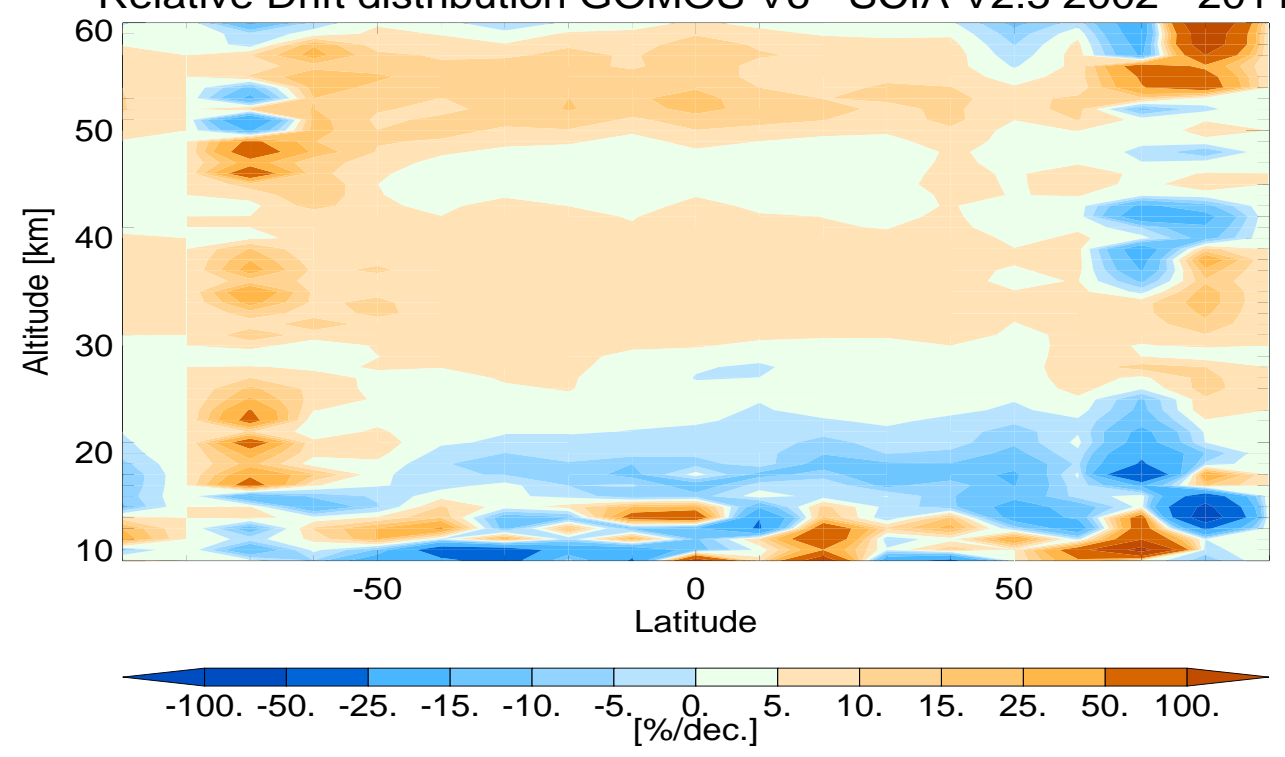

Figure 1: GOMOS - SCIAMACHY 


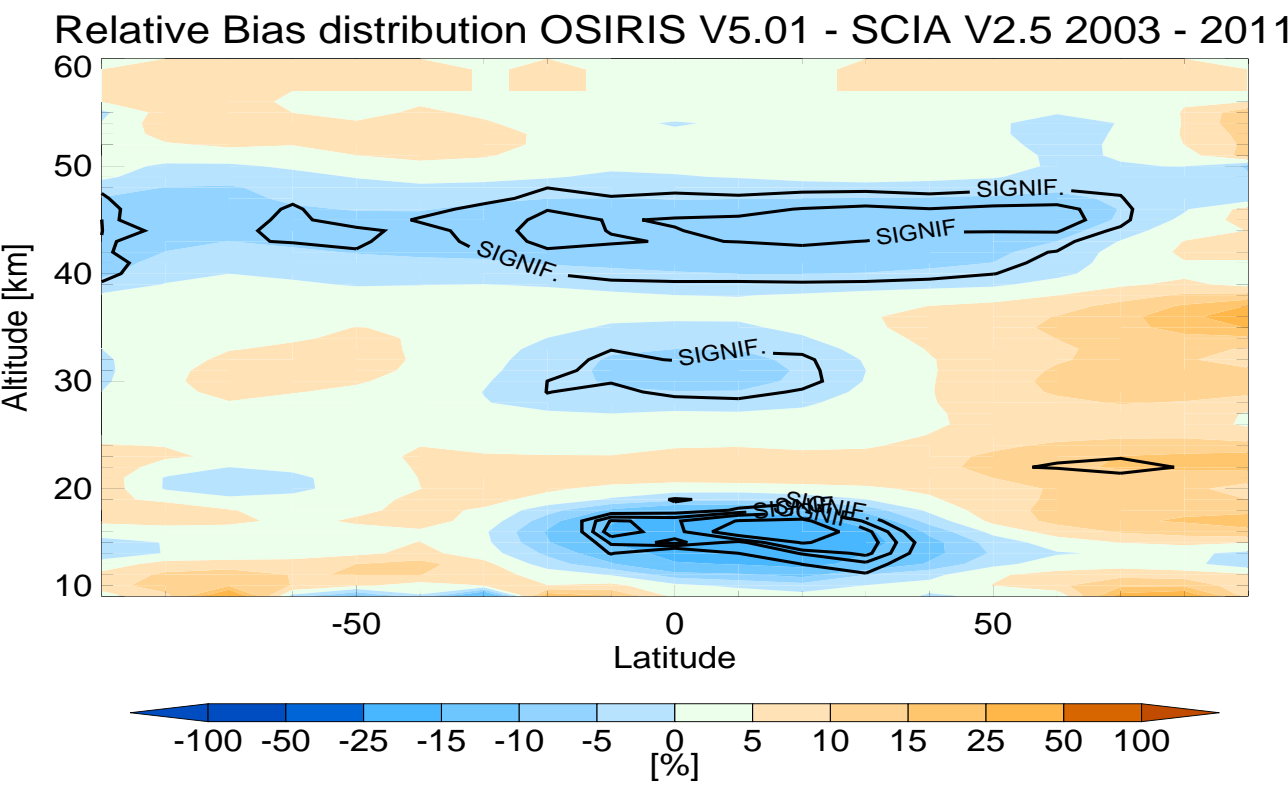

Relative Drift distribution OSIRIS V5.01 - SCIA V2.5 2003 - 2011

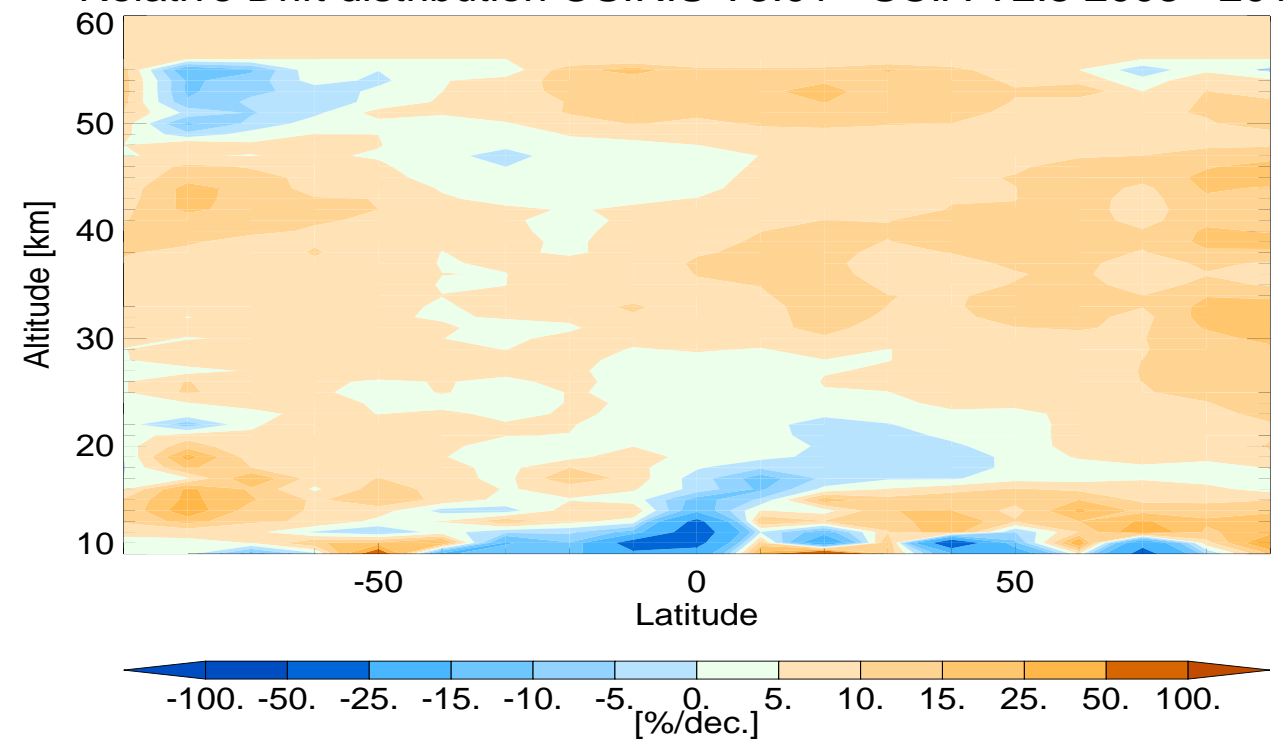

Figure 2: OSIRIS - SCIAMACHY 

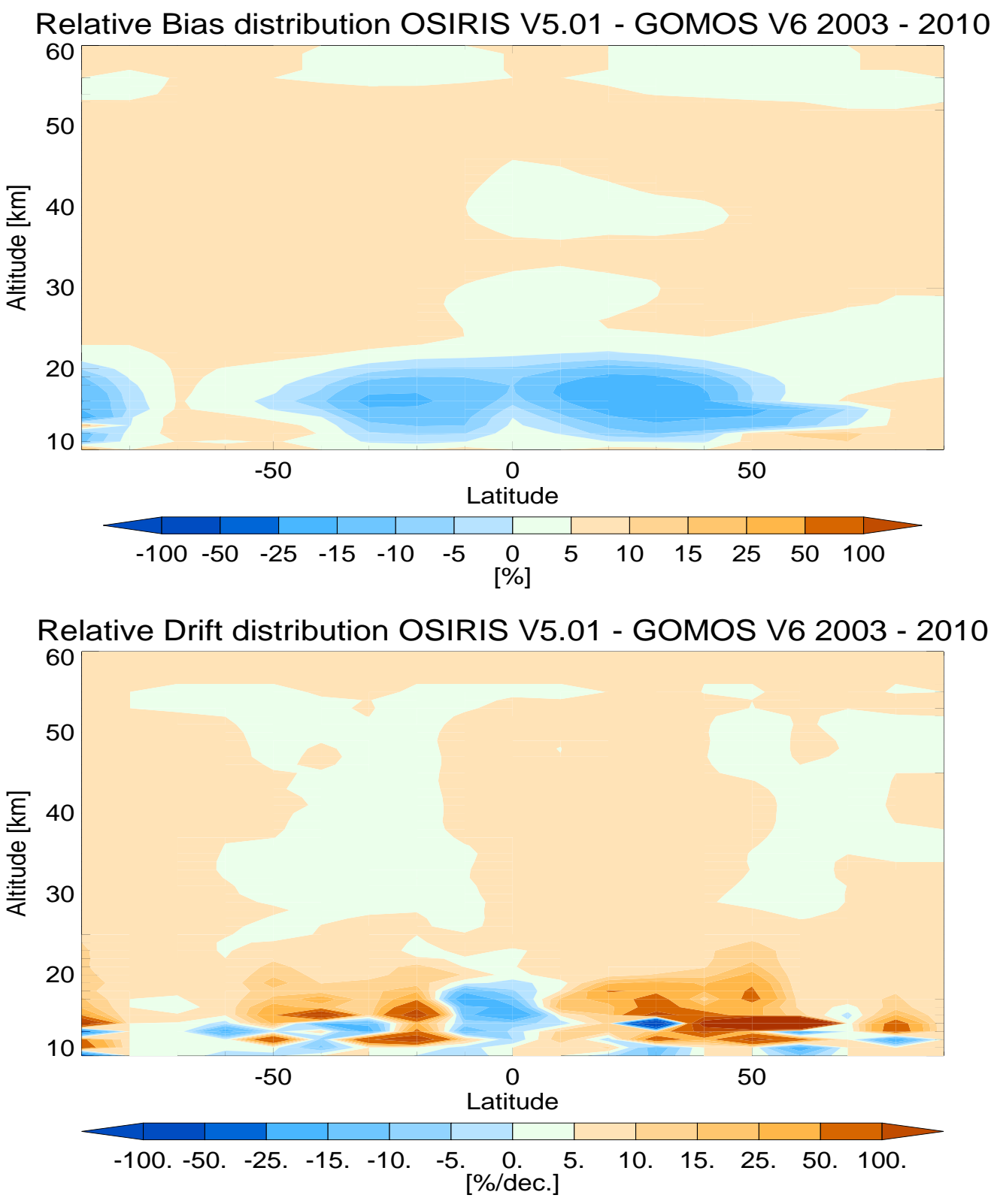

Figure 3: OSIRIS - GOMOS 
Relative Bias distribution MIPAS V5R_220 - SCIA V2.5 2005 - 2011
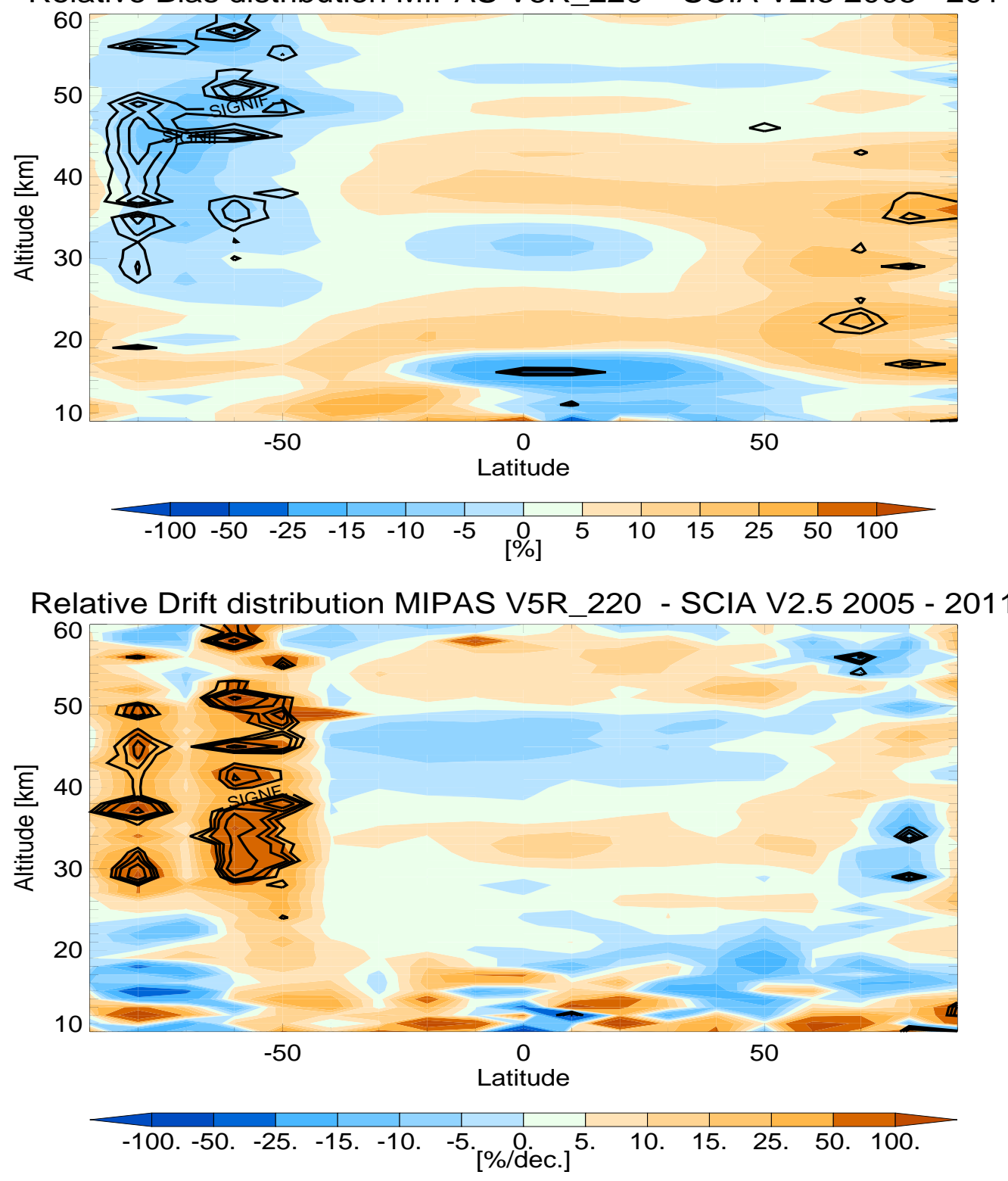

Figure 4: MIPAS - SCIAMACHY 

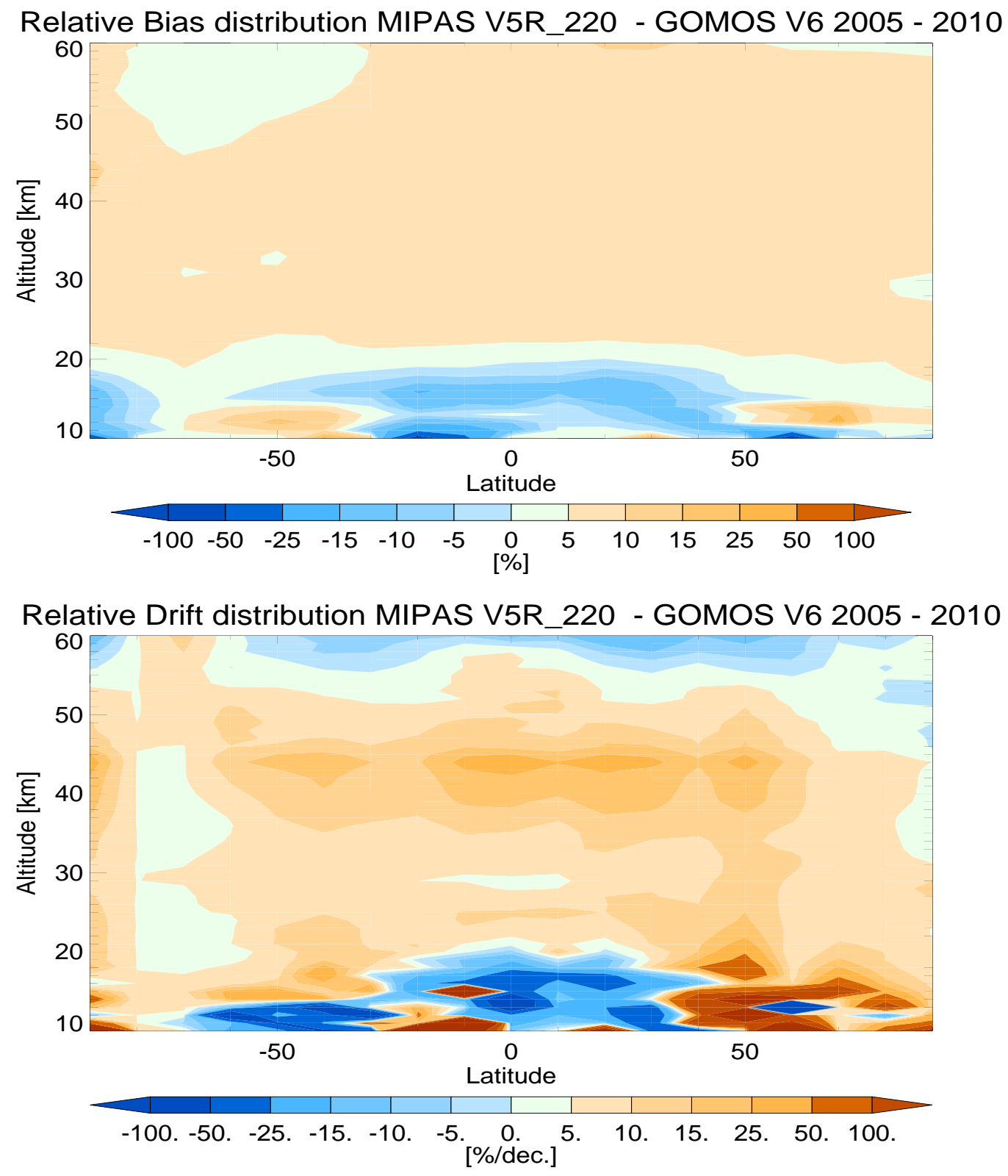

Figure 5: MIPAS - GOMOS 
Relative Bias distribution MIPAS V5R_220 - OSIRIS_V5.01 2005 - 2010
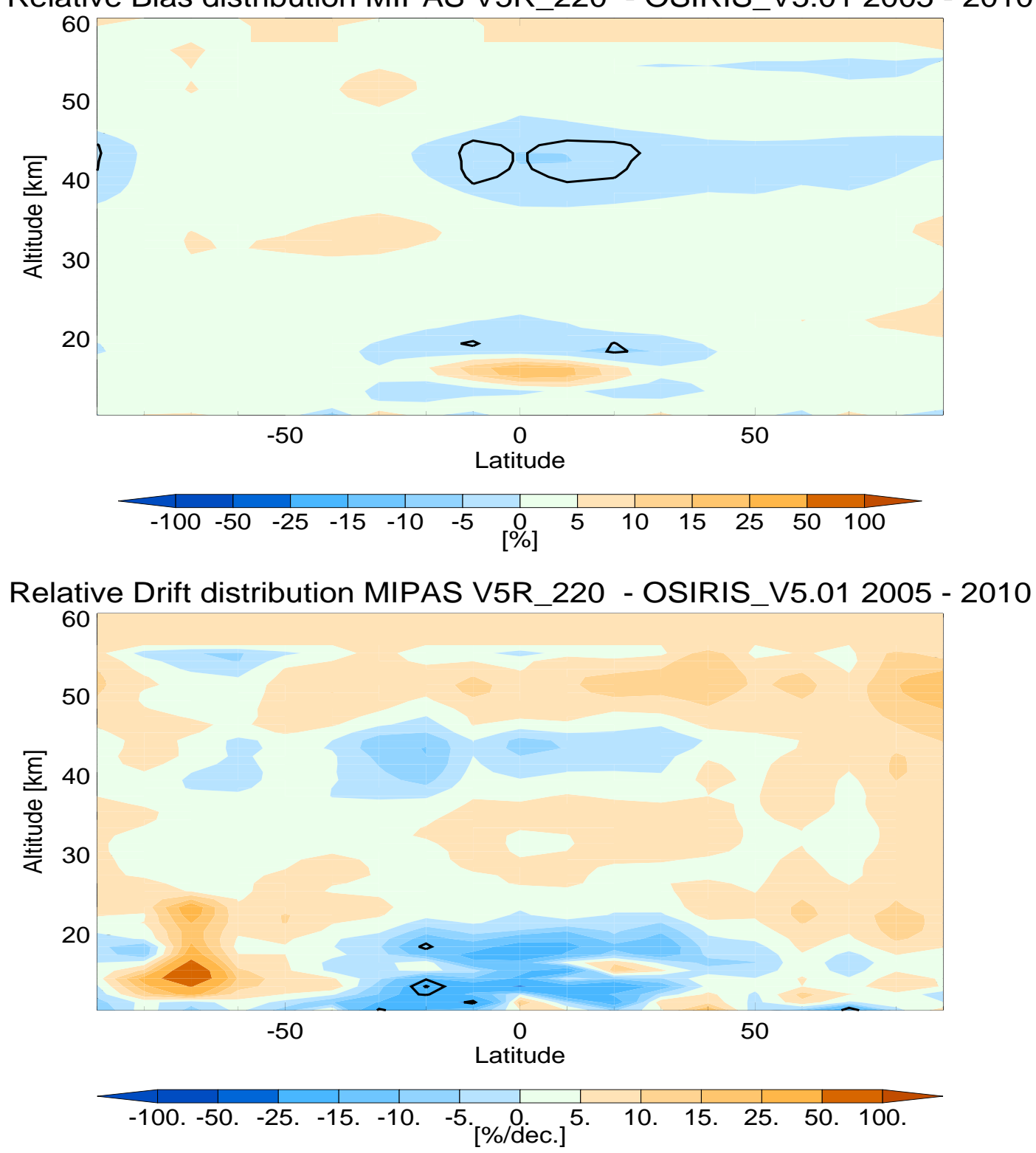

Figure 6: MIPAS - OSIRIS 
Relative Bias distribution ACE_FTS V3.0 - SCIA V2.5 2004 - 2010
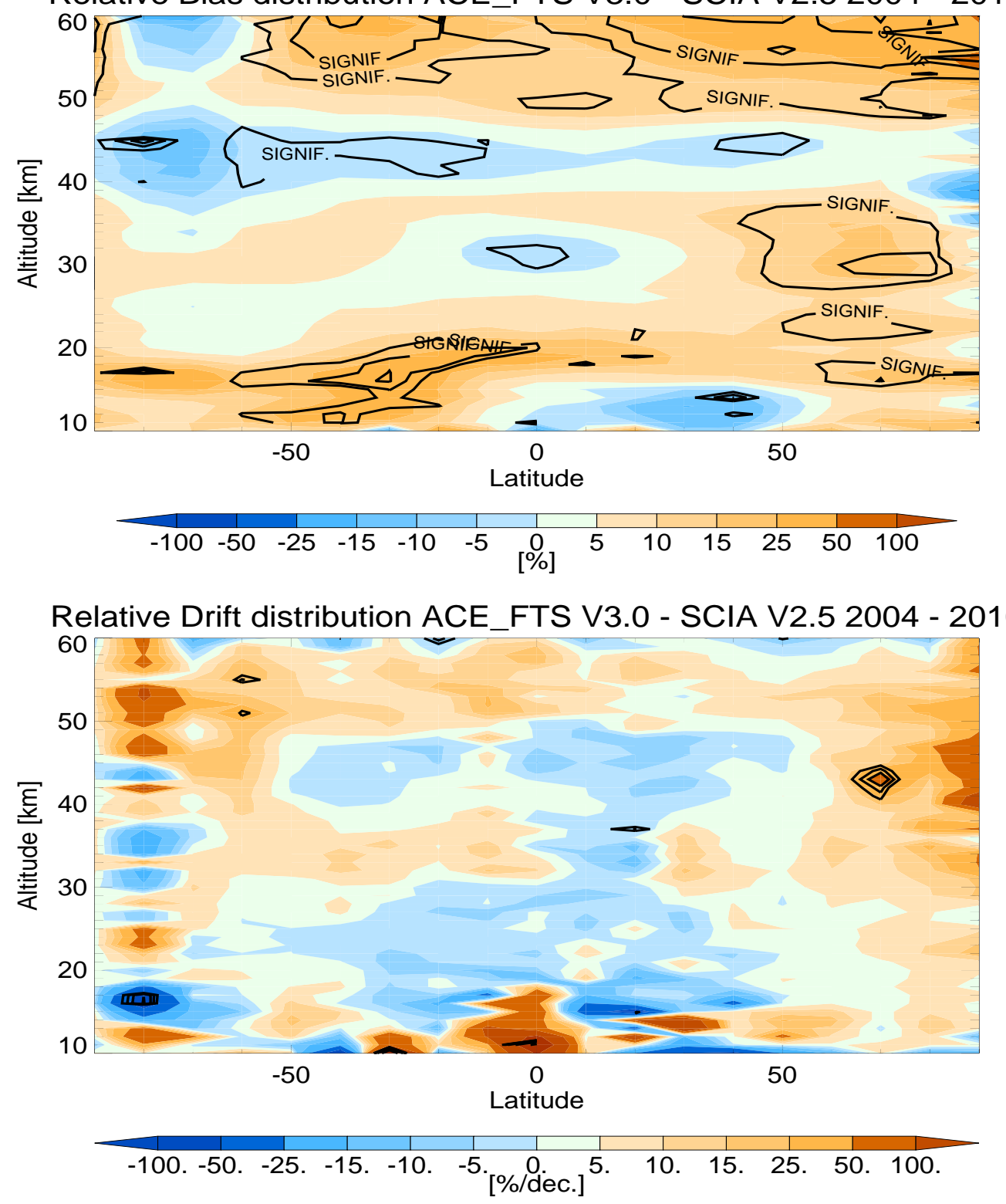

Figure 7: ACE FTS - SCIAMACHY 
Relative Bias distribution ACE V 3.0 - GOMOS V6 2004 - 2010 60

50

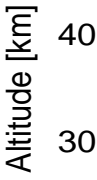

20

10

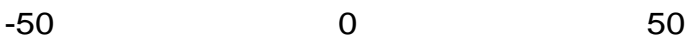

Latitude

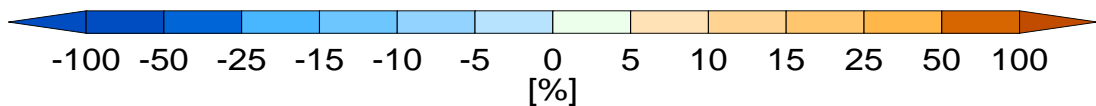

Relative Drift distribution ACE V 3.0 - GOMOS V6 2004 - 2010

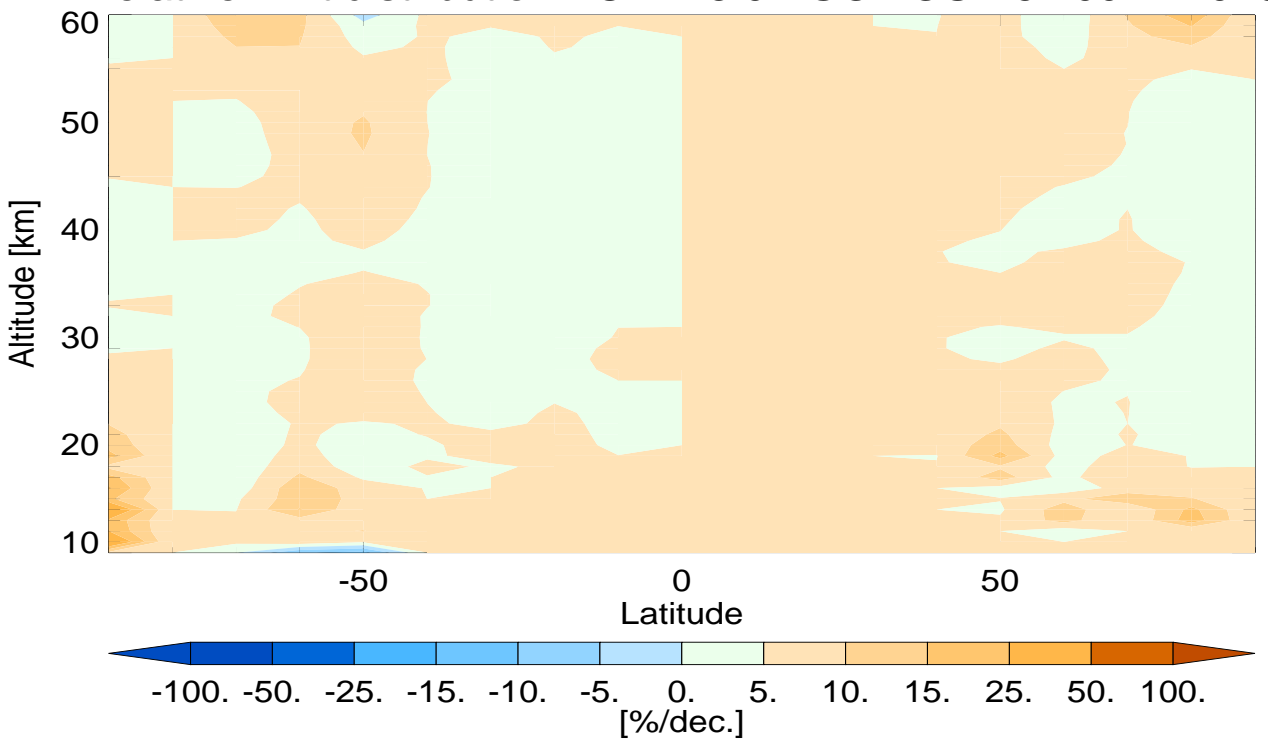

Figure 8: ACE FTS - GOMOS 
Relative Bias distribution ACE V 3.0 - OSIRIS_V5.012004- 2010
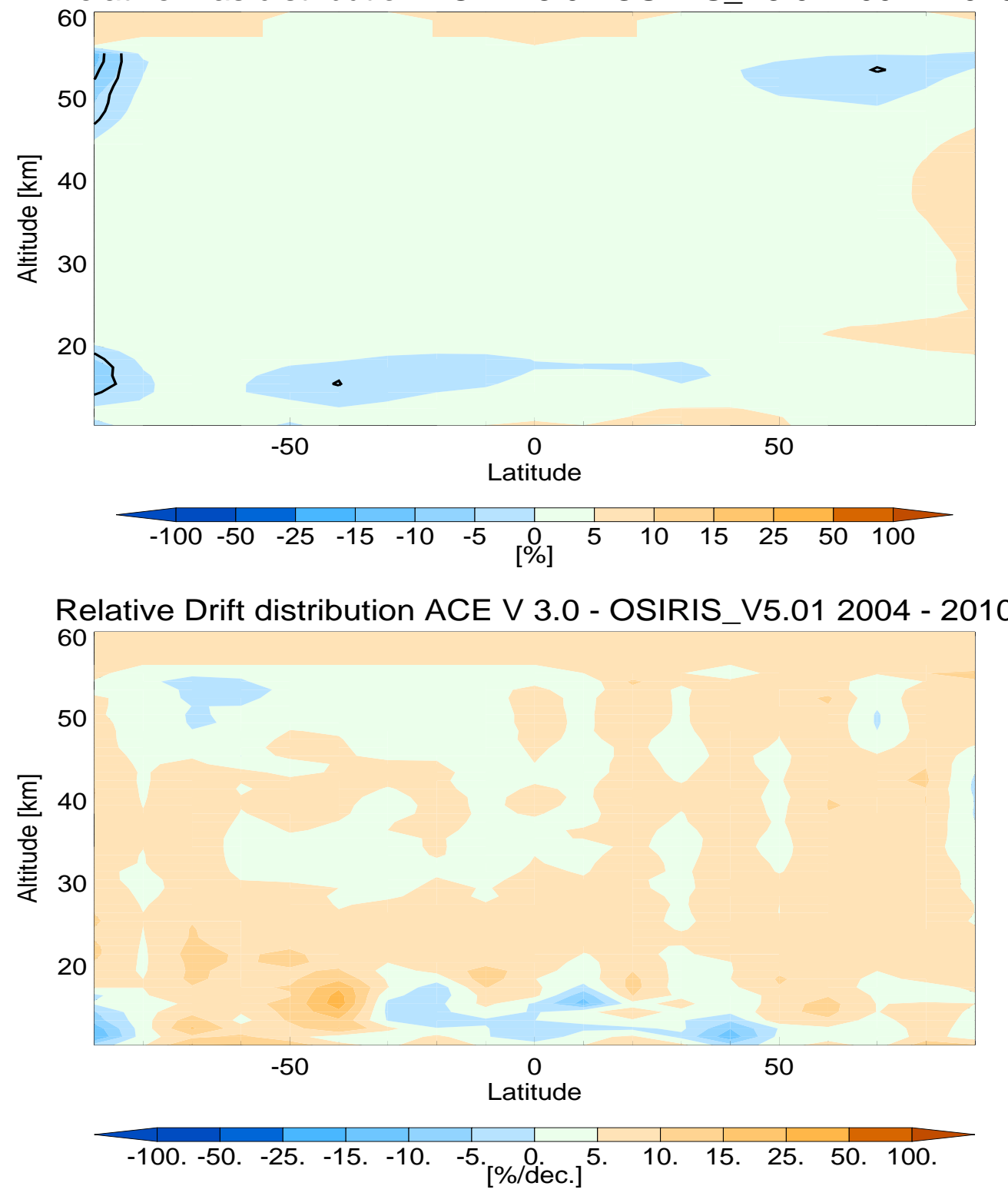

Figure 9: ACE FTS - OSIRIS 
Relative Bias distribution ACE V 3.0 - MIPAS_V5_R_220 2005 - 2010

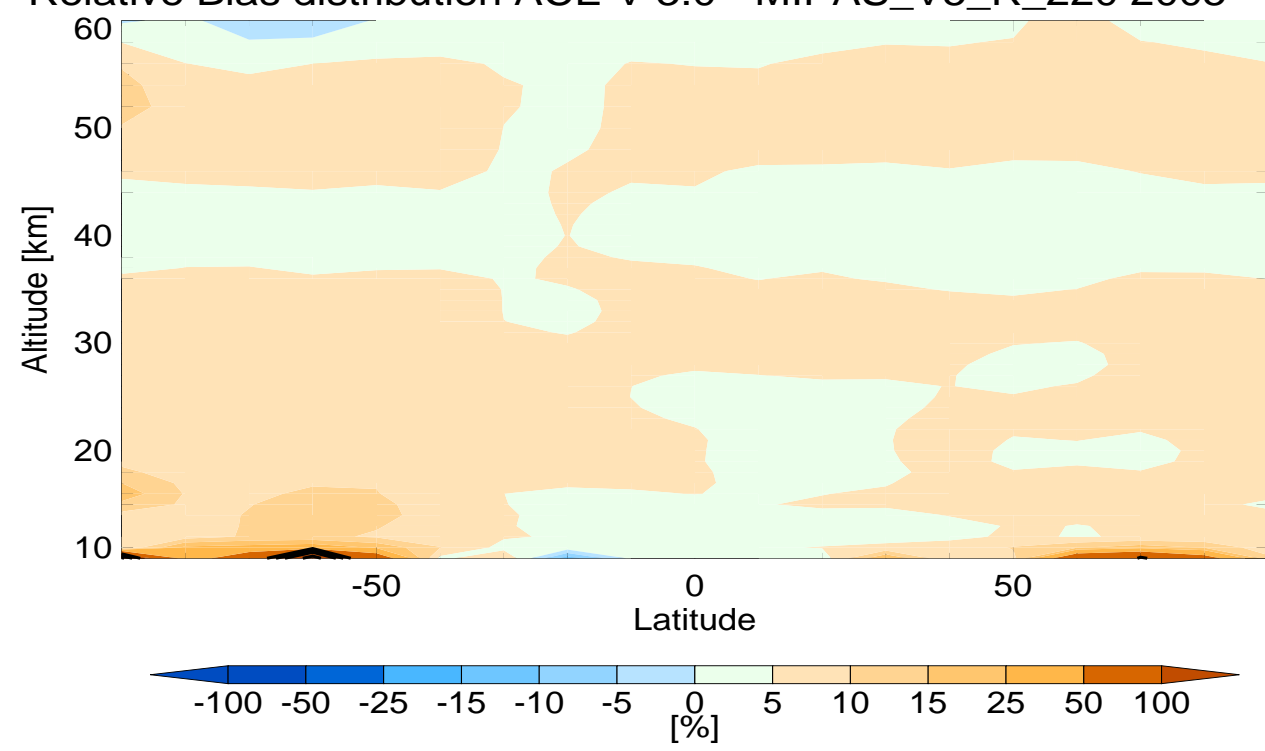

Relative Drift distribution ACE V 3.0 - MIPAS_V5_R_220 2005 - 2010

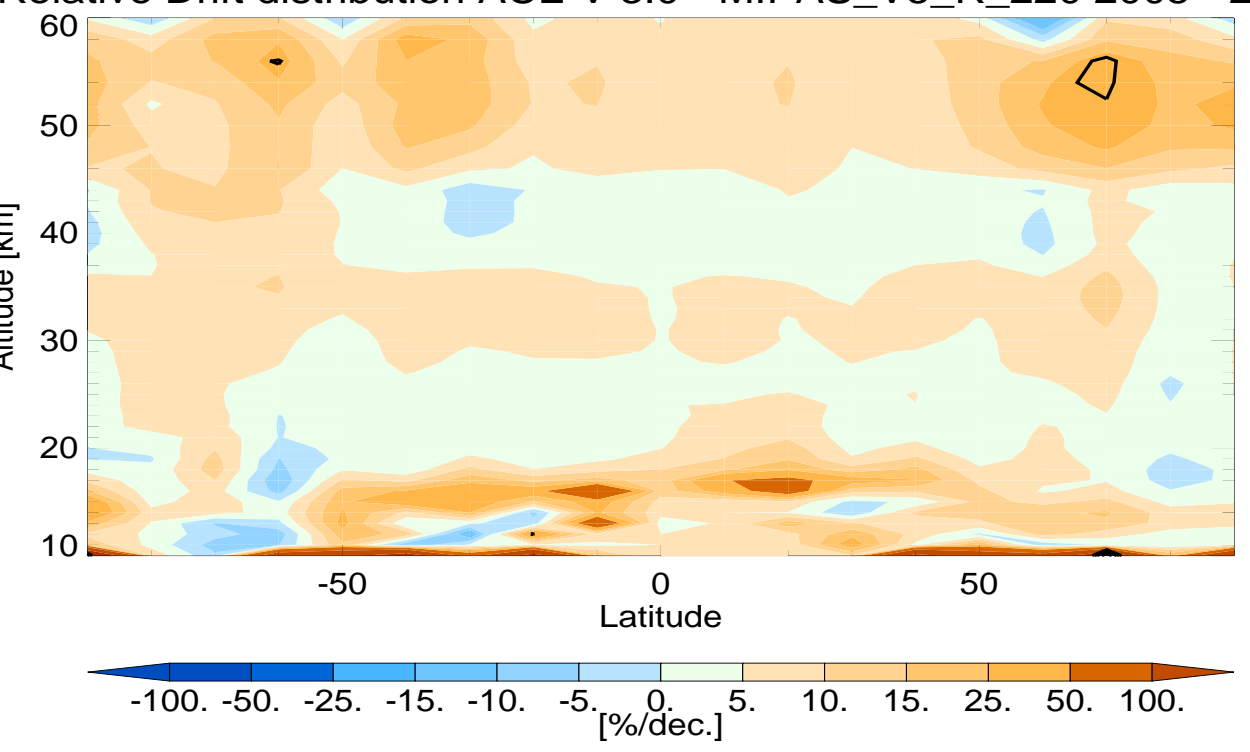

Figure 10: ACE FTS - MIPAS 
Relative Bias distribution SMR V2.1 - SCIA V2.5 2002 - 2010

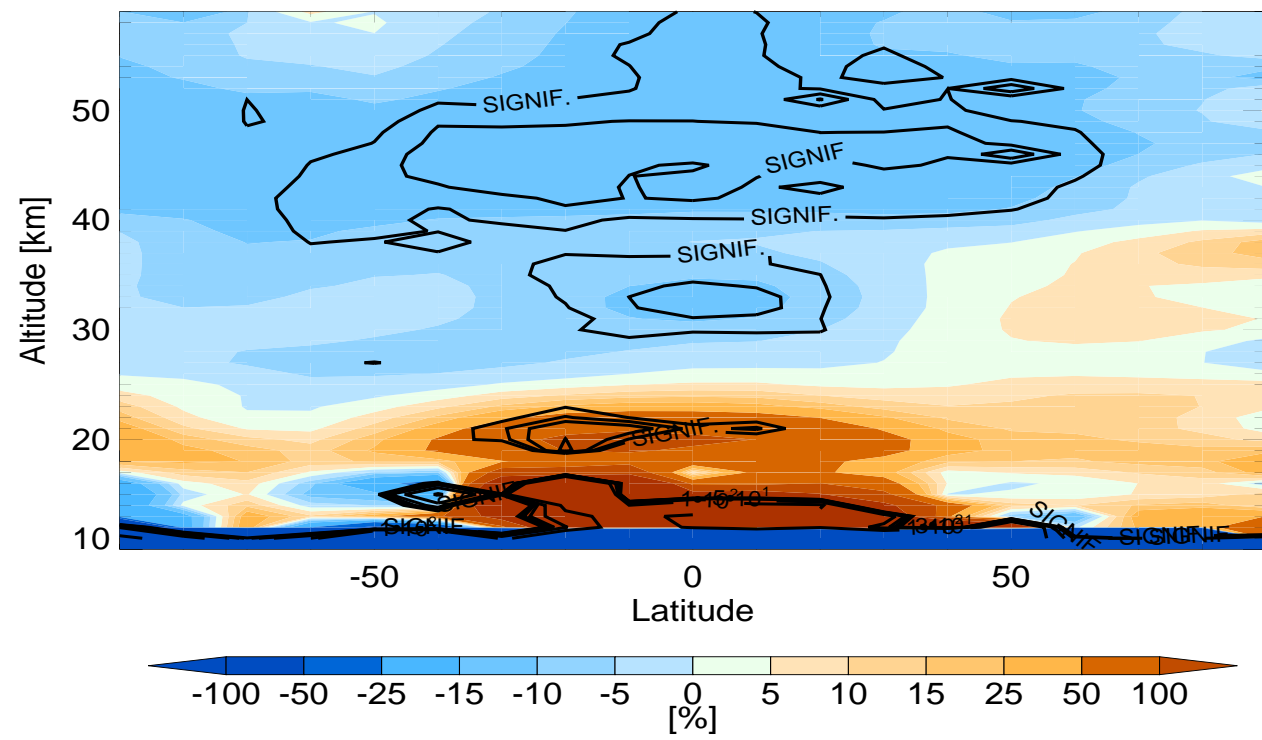

Relative Drift distribution SMR V2.1 - SCIA V2.5 2002 - 2010

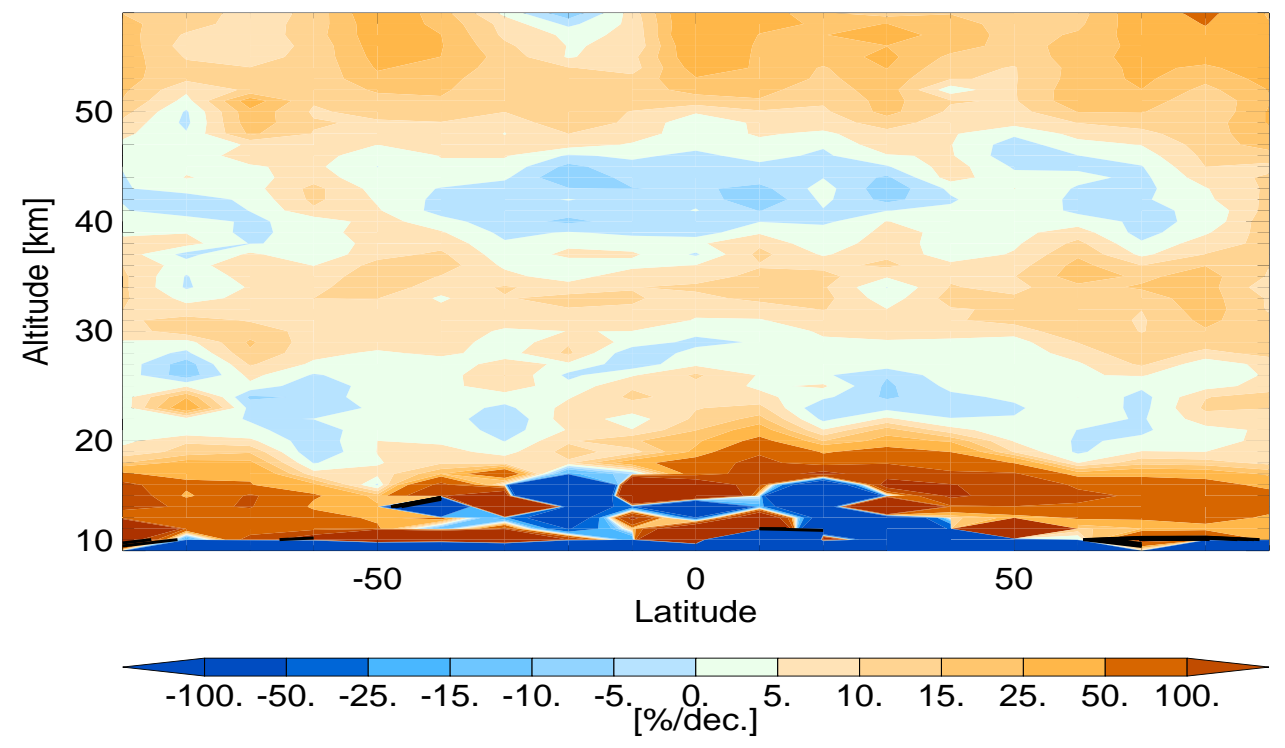

Figure 11: SMR - SCIAMACHY 
Relative Bias distribution SMR V2.1 - GOMOS V6 2002 - 2010

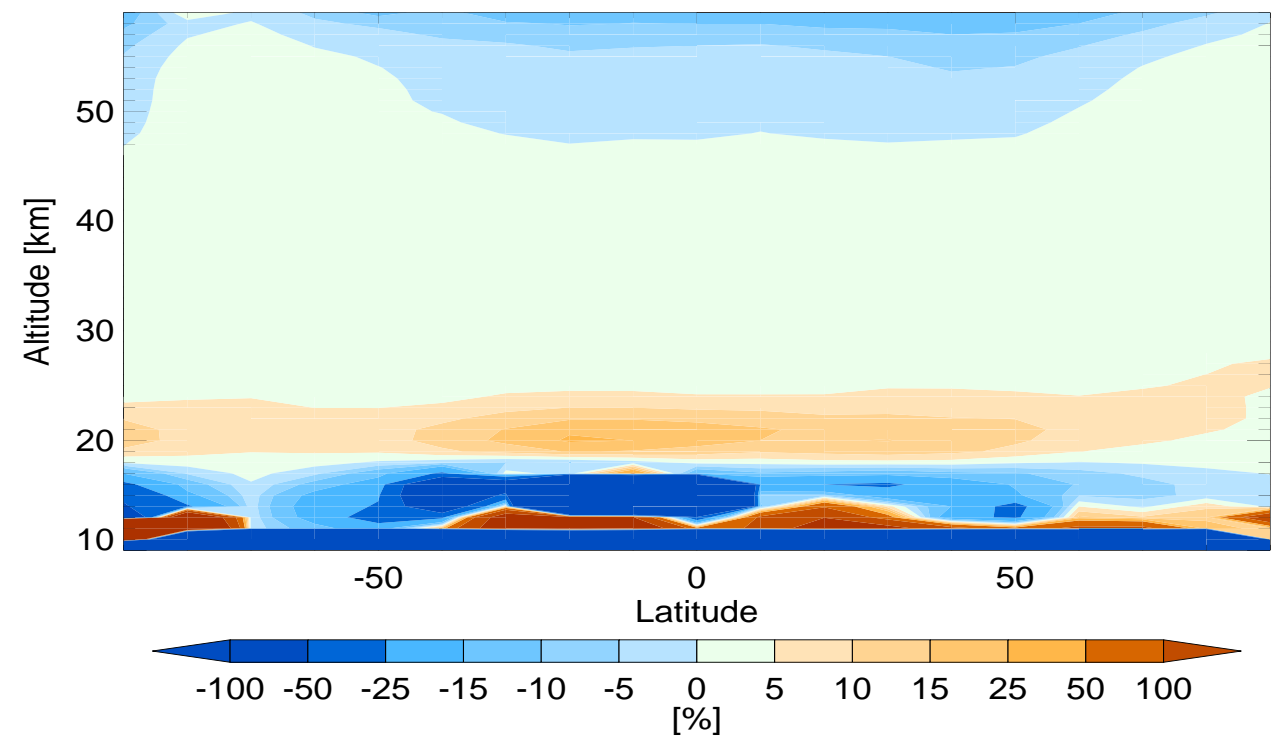

Relative Drift distribution SMR V2.1 - GOMOS V6 2002 - 2010

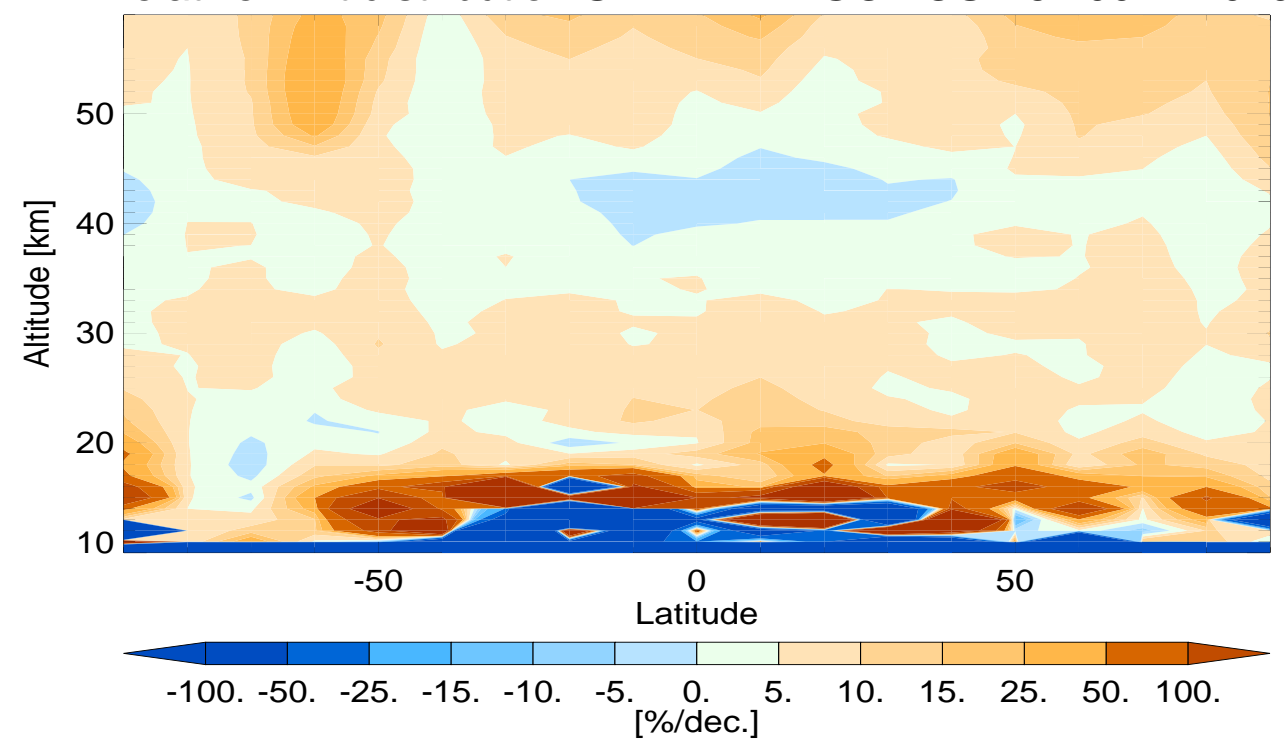

Figure 12: SMR - GOMOS 
Relative Bias distribution SMR V2.1 - OSIRIS_V5.01 2002 - 2010 60

$50)$

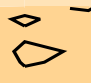

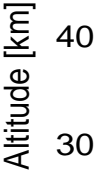
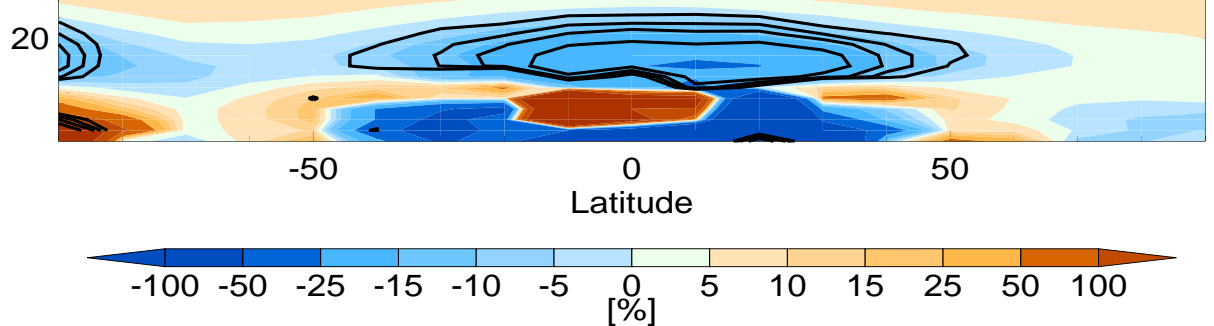

Relative Drift distribution SMR V2.1 - OSIRIS_V5.01 2002 - 2010

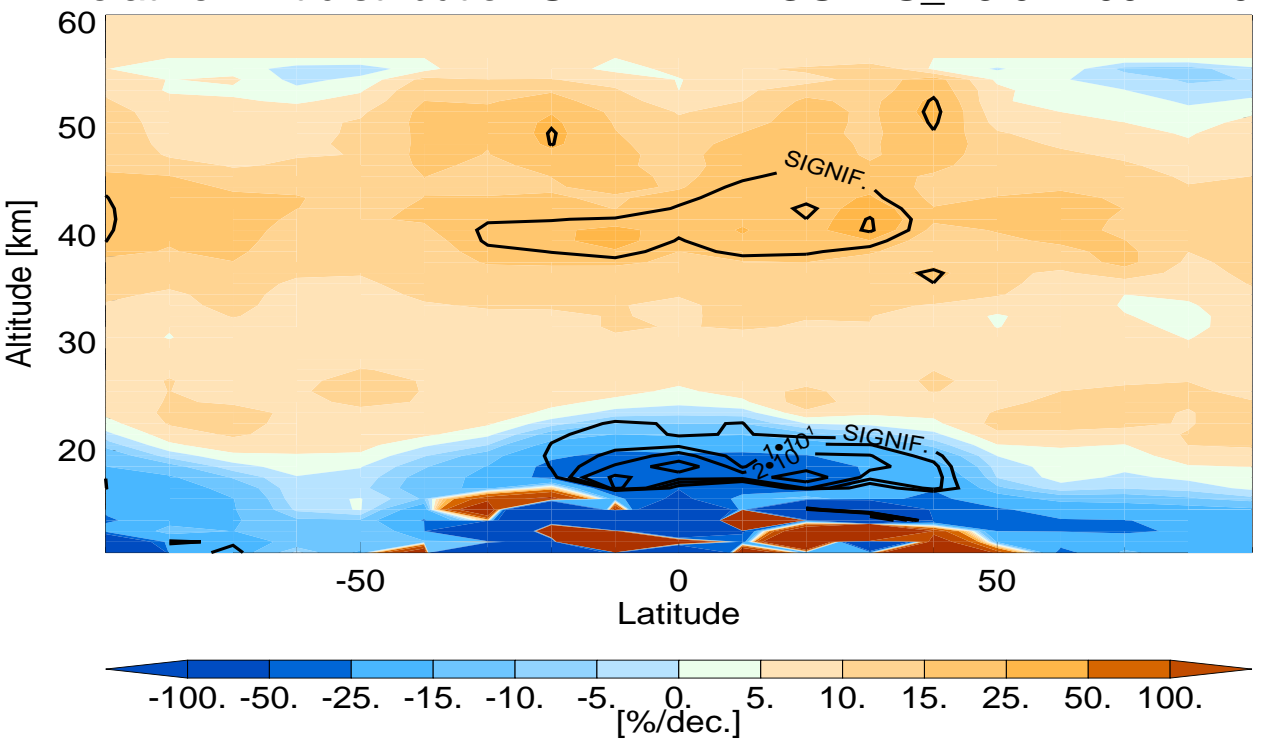

Figure 13: SMR - OSIRIS 

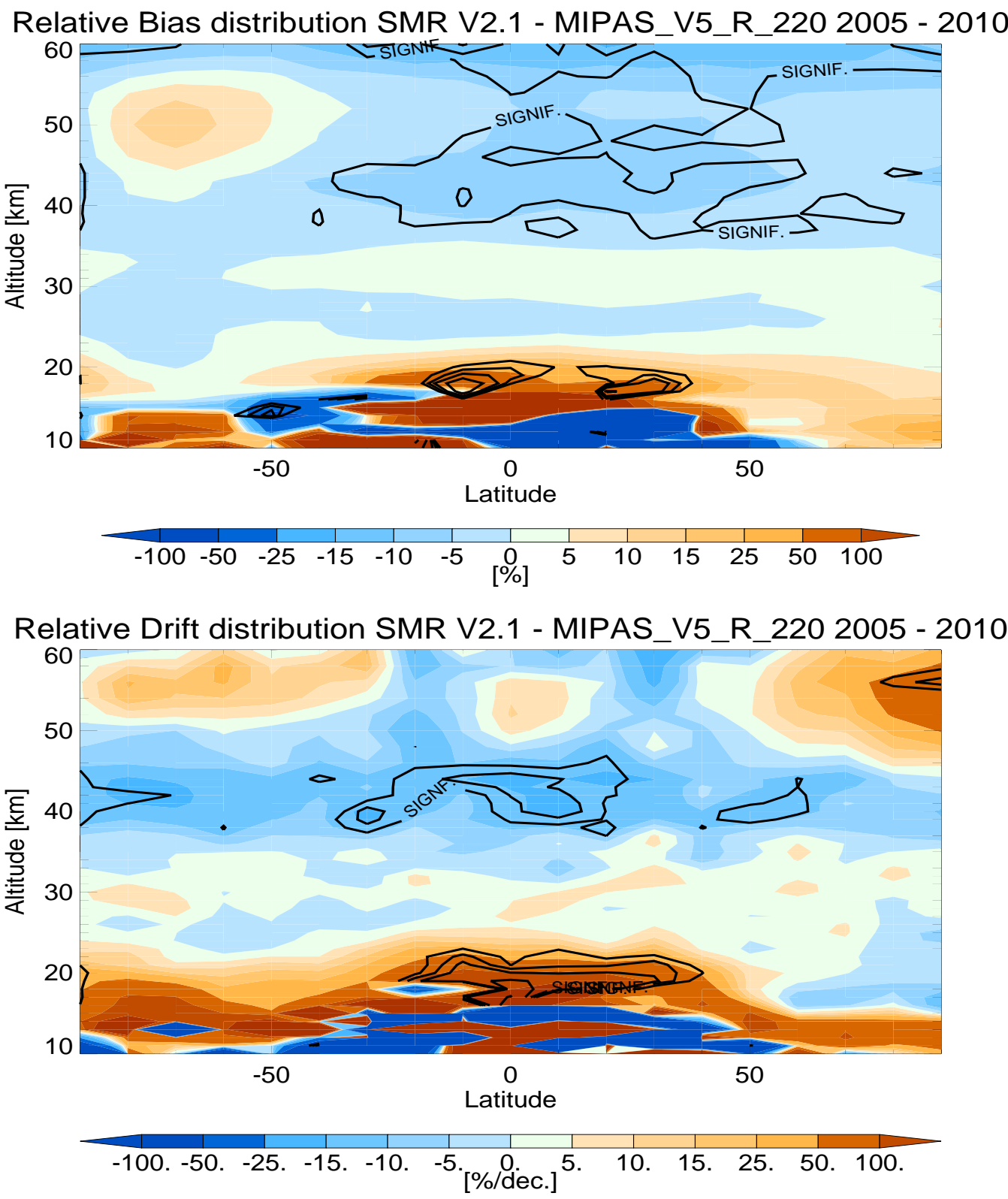

Figure 14: SMR - MIPAS 

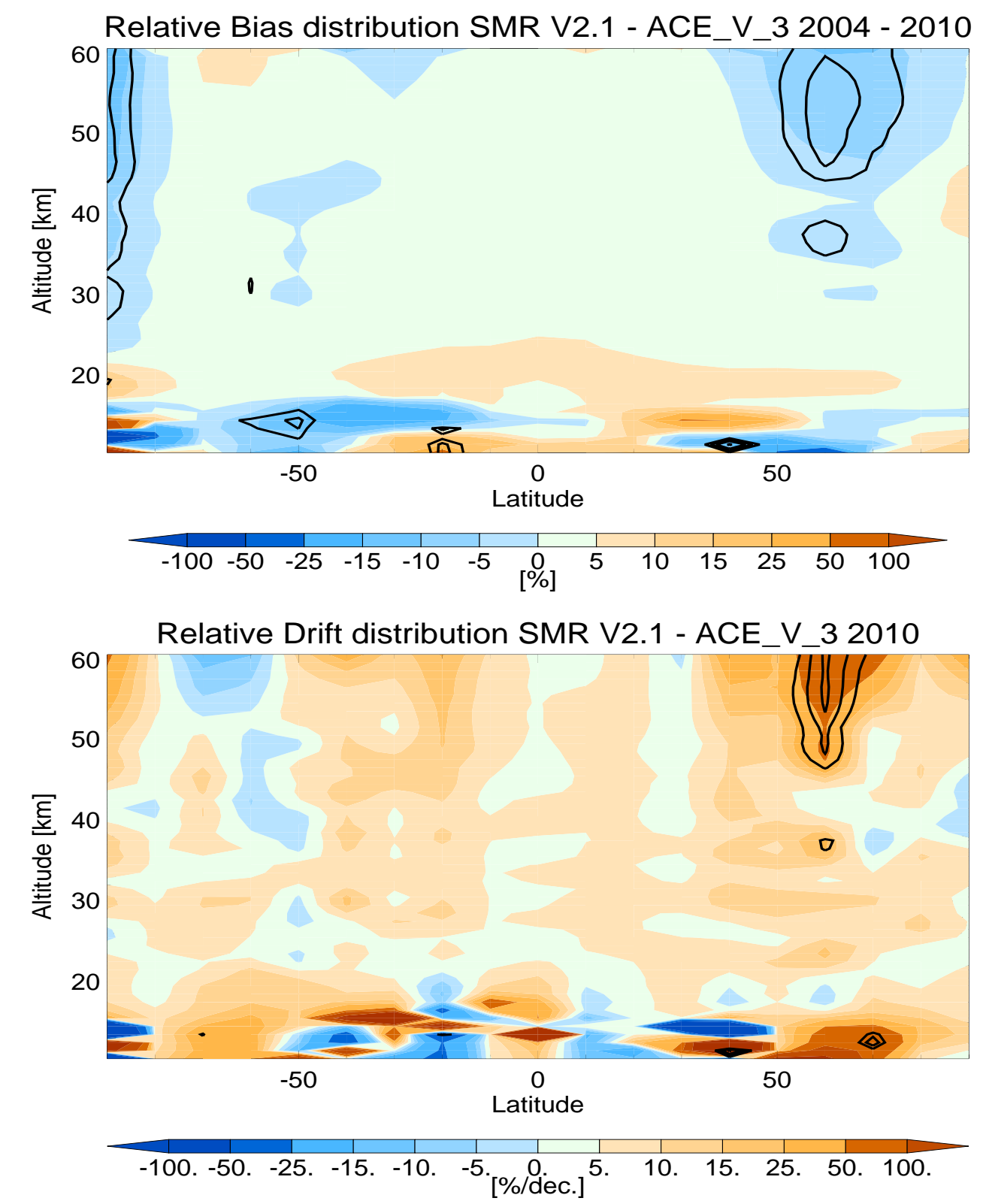

Figure 15: SMR - ACE FTS 